\title{
Exposure Time Relations for Kossel Microdiffraction Photographs
}

\author{
H. Yakowitz and D. L. Vieth
}

(April 23, 1965)

\begin{abstract}
The exposure time for a Kossel photograph may vary from a few seconds to a few hours. Therefore, it is desirable to be able to estimate the exposure time for various experimental conditions. Hence, semiempirical relations for the exposure time of a Kossel microdiffraction pattern have been developed. Equations are presented for both transmission and back reflection Kossel photographs. These equations are tested for validity using two different commercially available $\mathrm{x}$-ray films. It is shown that the agreement of actual exposure times with predicted exposure times is valid within 10 to 15 percent.
\end{abstract}

It has long been known that the exposure time of a Kossel photograph has a well-defined optimum [1]. ${ }^{1}$ However, useful analytical expressions enabling one to calculate the exposure time have not been presented. Therefore, the purpose of this paper is to propose expressions for the exposure time in both the transmission and the back reflection Kossel regions.

In the transmission mode, the Kossel conics usually appear light on a darker background and the contrast is, at best, not good $[2,3]$. In the back reflection region, the conics appear darker than the background, and the contrast may be somewhat better than in transmission [2]. The only practical source for Kossel patterns is a finely focused electron beam which is allowed to strike either the sample or a source foil in close proximity to the sample. The latter case will be discussed as it is the more general and more useful. It will be assumed that the film is in a vacuum and that once the $\mathrm{x}$ rays leave the sample, having undergone the usual exponential reduction in intensity, they travel unimpeded to the film. If an x-ray window and airpath intervene, the reduction in intensity of the $\mathrm{x}$ rays emergent from the sample can also be accounted for by the usual exponential retardation law which simply appears as a multiplier.

Using a focused beam of electrons, one may vary the specimen current, i.e., the number of electrons flowing to ground per unit time from specimen or foil, and the accelerating potential of the electrons. A knowledge of the number of photons per electron which strike the film and of the film area is necessary. Some provision for the fact that the exposure is not constant over the expanse of a flat film is also necessary. Bearing each of these requirements in mind, we may write for the exposure:

\footnotetext{
${ }_{1}^{1}$ Figures in brackets indicate the literature references at the end of this paper.
}

$$
\begin{aligned}
E=(60 t) & \frac{\left(i_{s}\right)}{(1-r)}(G)\left(\frac{\Omega_{s}}{A_{F}}\right) \\
& {\left[\frac{\left(n_{K}\right)_{f}}{4 \pi}\right] 6.25 \times 10^{12}\left[\exp \left[-\left(\bar{\mu}_{f} x_{f}+\bar{\mu}_{s} x_{s}\right)\right]\right], }
\end{aligned}
$$

where

$E$ is the exposure in photons $/ \mathrm{cm}^{2}$.

$i_{s}$ is the specimen current in microamperes.

$G$ is a factor accounting for variation of exposure with position on the flat film.

$\Omega_{s}$ is the solid angle subtended by the spherical segment containing the film.

$A_{F}$ is the exposed film area in $\mathrm{cm}^{2}$.

$\left(n_{K}\right)_{f} / 4 \pi$ is the total number of $K$ photons produced per unit solid angle by the foil per incident electron.

$6.25 \times 10^{12}$ is the number of electrons per microcoulomb of charge.

$r$ is the electron backscatter coefficient of the foil [4].

$\bar{\mu}_{f}$ and $\bar{\mu}_{s}$ are the equivalent linear absorption coafficients for the total spectrum produced in the foil and the sample respectively in $\mathrm{cm}^{-1}[1]$.

$x_{f}$ and $x_{s}$ are the thicknesses in $\mathrm{cm}$ of the foil and sample respectively.

$t$ is the exposure time in minutes.

Values of $n_{0} \equiv\left[\left(n_{K}\right)_{f} / 4 \pi\right]$ as a function of atomic number and the accelerating potential of the electrons are available in the literature $[5,6,7]$. The area of the film can be found by reference to figure 1 as:

$$
A_{F}=\pi D^{2} \tan ^{2} \eta,
$$

where

$D$ is the source to film distance in $\mathrm{cm}$;

$\eta$ is the half-cone angle subtended by the film. 
The values of $D$ and $\eta$ are usually well known.

The inclusion of $G$ arises from the fact that the exposure will be greatest at the film center and least at the edge. This is a combination of two effects, the first of which is an increasing absorption path within the sample with increasing angle of emergence. The other effect is that of the geometry, i.e., a unit of solid angle subtends a larger area on the film at the edge than at the center. It has been observed that the net effect is a slowly decreasing function of the angle $\eta$. This can be suitably approximated by defining $G$ as :

$$
G \equiv\left[\frac{1+\cos \eta}{2}\right]
$$

Over the usual range of $\eta$ values, i.e., $20^{\circ} \leq \eta \leq 36^{\circ}$, the inclusion of this form of $G$ increases the exposure time 3 to 10 percent. It is interesting to note that the important central film area in figure 1 defined by the angle $\eta / 2$ and the ray, $Q$, will be nearly uniformly blackened.

The factor $\frac{i_{s}}{1-r}$ is

the beam current, i.e., the incident electron current. It is for this current that $n_{0}$ values have been derivad. The simple value " $r$ " can be used since the effect on the specimen current, $i_{s}$, due to secondary electrons which may be generated is quite small.

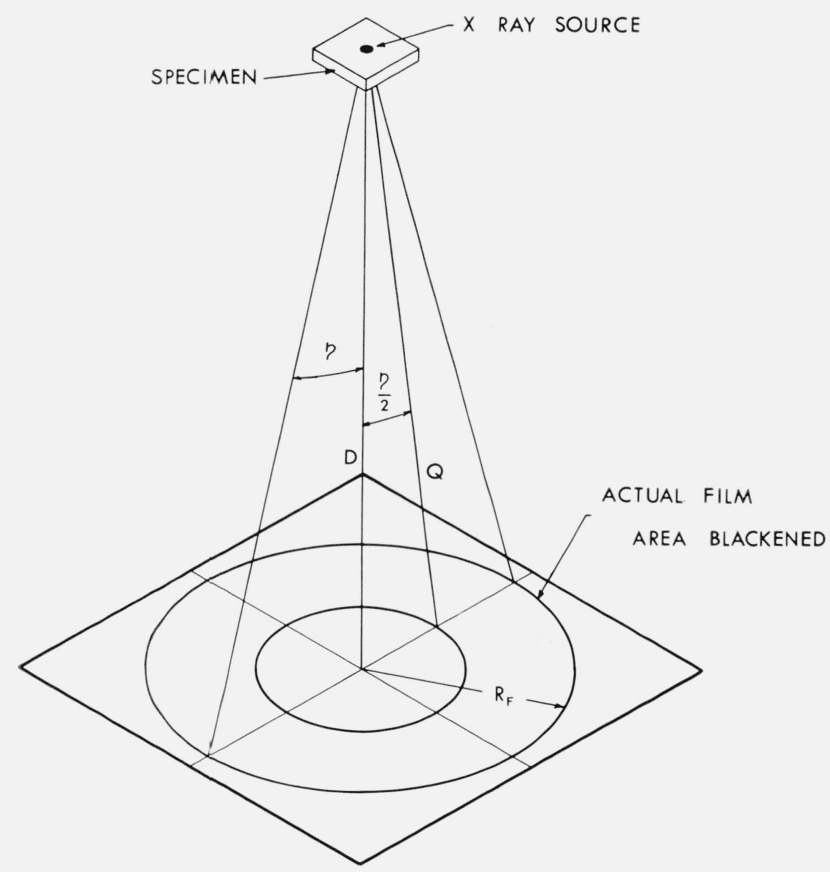

Figure 1. Geometrical relations between the $x$-ray source and the film.
The solid angle subtended by the spherical segment containing the film is given by [8]:

$$
\Omega_{s}=2 \pi(1-\cos \eta)
$$

Thus, $\left(n_{0} \Omega_{s}\right)$ is a measure of the number of photons per incident electron which would actually fall on the film if there were no exponential absorption.

The value $\bar{\mu}$ can be approximated by the relation $\bar{\mu} \approx \% 3 \mu, \mu$ being the linear absorption coefficient for $K_{\alpha}$ or $L_{\alpha}$, for excitation over-voltage ratios of 3 to 4 [3] and for most $\mu$ values used in Kossel analysis. The value of $x_{f}$ should be kept as small as practical. The value of $x_{s}$ should be the approximate optimum thickness for the transmission case [3]. In back reflection, an effective depth can be taken as an approximation to $x_{s}$. This point will be discussed later.

Equation (1) may now be rewritten as:

$$
\begin{aligned}
E=(t) \frac{\left(i_{s}\right)}{(1-r)} \frac{1+\cos \eta}{2} 2 \pi \frac{(1-\cos \eta) r_{s_{0}}}{\pi D^{2} \tan ^{2} \eta} & \left(3.75 \times 10^{14}\right)\left\{\exp -\left[\frac{2}{3}\left(\mu_{f} x_{f}+\mu_{s} x_{s}\right)\right]\right\} .
\end{aligned}
$$

Solving for $t$ we obtain:

$$
t(\min )=\frac{2.67 \times 10^{-15} E\left(\sec ^{2} \eta\right) D^{2}}{n_{0} i_{s} \quad(1-r)} \quad\left\{\exp -\left[\frac{2}{3}\left(\mu_{f} x_{f}+\mu_{s} x_{s}\right)\right]\right\} .
$$

It is necessary to obtain a value for $E$ empirically for each type of film to be used. This $E$ value, henceforth called $E_{0}$, is that exposure density yielding the maximum contrast between the Kossel conics and background for a given film at a given distance, $D$. Jt is emphasized that the $E_{0}$ value in eq (6) represents a compromise between the exposure at the extreme edges of the film and the exposure at the film center. It is to be expected that the exposure density $E_{0}$ will be essentially constant for a given film type, independent of other camera parameters.

Equation (6) as written is strictly speaking only applicable to transmission Kossel photographs. In the back reflection region, precisely the same relation holds true except that $x_{s}$ is undefined. However, a reasonably good approximation to $x_{s}$ can be made.

Such an approximation can be made following Il'in's treatment [9]. Il'in proposes an effective thickness, $d_{f}$, of the absorbing layer chosen such that the function $\exp -\left(\mu d_{f}\right)$ would give total attenuation of an analytic line such as $K \alpha_{1}$ or $L \alpha_{1}$, in the working volume of the specimen, i.e., at $\eta=90^{\circ}$. It is presumed that

$$
\rho d_{f}=H
$$

in which $\rho$ is the density of the specimen and $H$ is a constant for a given $\eta$ value. As support for eq (7) the fact that $d_{f}$ is related to the total thickness of the emitting portion of the target is invoked [9]. 
According to the Whiddington law, this thickness is determined for a given accelerating potential by the density of the emitter.

By rearranging Il'in's relations [10] and invoking electron probe microanalyzer results presented in the literature [11-14], it is possible to plot an $H$ versus $\eta$ curve. This plot is shown in figure 2 .

Noting that $x_{\varepsilon}$ is to be replaced by $d_{f}$ in eq (6) for back reflection cases, one obtains:

$$
\mu_{s} x_{s}=\mu_{s} d_{f}=H(\mu / \rho)_{s} .
$$

Hence for back reflection, eq (6) becomes

$$
\begin{aligned}
& t(\min )=\frac{2.67 \times 10^{-15} \mathrm{E}\left(\mathrm{sec}^{2} \eta\right) D^{2}}{\left(n_{0}\right)\left(i_{s}\right)}(1-r) \\
& \left\{\exp -\frac{2}{3}\left[\mu_{s} x_{s}+H(\mu / \rho)_{s}\right]\right\}
\end{aligned}
$$

In order to test the validity of eqs (6) and (9), the value of $E$ was calculated for two different film types commonly used in the preparation of Kossel microdiffraction photographs. One of these was moderately coarse-grained duplitized film ${ }^{2}$ while the other was a moderately fine-grained single emulsion film. ${ }^{3}$ These shall be designated I and II respectively.

Using type I film for about $200 \mathrm{Fe}-\mathrm{K}$ radiation exposures of $\mathrm{Fe}-3 \mathrm{w} / \mathrm{o} \mathrm{Si}$ alloy, it was found that the value of $E_{\mathrm{I}}$ was $1.6 \times 10^{8}$ photons $/ \mathrm{cm}^{2}$ for high contrast, high resolution transmission photographs [15].

For type II film, the exposure time versus iron thickness curve given by Morris and Ogilvie [16] was used to calculate $E_{\mathrm{II}}$. The value obtained was $1.4 \times 10^{9}$ photons $/ \mathrm{cm}^{2}$. Inserting the values of $E_{\mathrm{I}}$ and $E_{\mathrm{II}}$ into eq $(6)$ we obtain:

$$
\begin{aligned}
t_{\mathrm{I}}(\min )=\frac{4.1 \times 10^{-7} D^{2}\left(\sec ^{2} \eta\right)}{\left(i_{s}\right)\left(n_{0}\right)}(1-r) \\
\left\{\exp -\left[\frac{2}{3}\left(\mu_{f} x_{f}+\mu_{s} x_{s}\right)\right]\right\} .
\end{aligned}
$$

$$
\begin{aligned}
& t_{\mathrm{II}}(\min )=\frac{4.0 \times 10^{-6} D^{2}\left(\sec ^{2} \eta\right)}{\left(i_{s}\right)\left(n_{0}\right)}(1-r) \\
&\left\{\exp -\left[\frac{2}{3}\left(\mu_{f} x_{f}+\mu_{\circ} x_{s}\right)\right]\right\} .
\end{aligned}
$$

It remained to check eqs (10) with independent data. For type I film, Gielen's exposure of germanium with $\mathrm{Cu}-\mathrm{K}$ radiation was used [17]. The required parameters were:

$D=6 \mathrm{~cm}$

$\eta=20.3^{\circ}$

$i_{s}=0.11 \mu \mathrm{A}$

$n_{0}=2.2 \times 10^{-4}$ photons/electron/unit solid angle at $30 \mathrm{keV}[7]$

$x_{f}=17.8 \times 10^{-4} \mathrm{~cm}$

$\mu_{f}=455 \mathrm{~cm}^{-1}$

$x_{s}=1.52 \times 10^{-2} \mathrm{~cm}$

$\mu_{s}=385 \mathrm{~cm}^{-1}$

$r_{\mathrm{Cu}}=0.29$.

2 Kodak A A film.

3 Kodak M film,

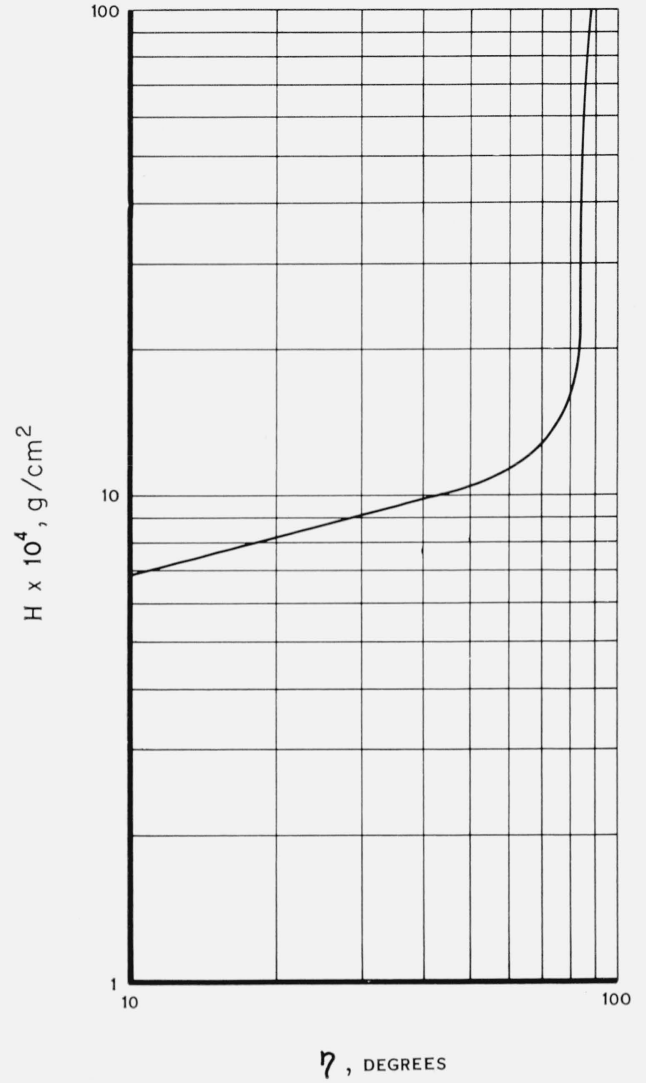

Figure 2. The value of $\mathrm{H}$ as a function of the half-angle of the film, $\eta$.

Gielen used a camera with an aluminum foil lighttight cap. The $(\mu x)$ value of the foil was 0.20 . The camera operated in vacuum. Using these data, eq (12a) predicted an exposure time of $49 \mathrm{~min}$. The actual exposure time for a high-contrast photograph was $50 \mathrm{~min}[18]$.

In order to check both eqs (9) and (10b), a back reflection photograph of iron using $\mathrm{Fe}-\mathrm{K}$ radiation taken on type II film was used as a model [19]. The required parameters were

$$
\begin{aligned}
D= & 12.4 \mathrm{~cm} \\
\eta= & 26^{\circ} \\
\dot{i}_{s}= & 2.0 \mu \mathrm{A} \\
n_{0}= & 6 \times 10^{-4} \text { photons/electron/unit solid angle } \\
& \quad \text { at } 40 \mathrm{keV}[6]
\end{aligned}
$$

$$
\begin{aligned}
x_{f} & =0 \\
(\mu / \rho)_{F_{e}}^{F_{e}} & =71.4 \\
H & =8.5 \times 10^{-4} \text { at } \eta=26^{\circ} \\
r_{F e} & =0.27 .
\end{aligned}
$$

Using these data, the predicted exposure time is $0.47 \mathrm{~min}$ or $28 \mathrm{sec}$. The actual time was $25 \mathrm{sec}$ [19]. 
It is perhaps of interest to comment that for a given set of conditions a simple equation results. For example, using the equipment parameters given for the iron exposure in back reflection, one obtains:

$$
\begin{aligned}
& t_{\mathrm{I}}=\frac{7.8 \times 10^{-5}}{\left(n_{0}\right)\left(i_{s}\right)}(1-r) \exp \left[5.7 \times 10^{-4}(\mu / \rho)_{s}+0.67 \mu_{f} x_{f}\right] \\
& t_{\mathrm{II}}=\frac{7.4 \times 10^{-4}}{\left(n_{0}\right)\left(i_{s}\right)}(1-r) \exp \left[5.7 \times 10^{-4}(\mu / \rho)_{s}+0.67 \mu_{f} x_{f}\right]
\end{aligned}
$$

It has been shown that eqs (6) and (9) represent the correct form of exposure time relations for Kossel photography. Because of the approximations made, e.g., for $\bar{\mu}$ and $d_{f}$, the uncertainties in absorption coefficients themselves, and possible inherent sample limitations [1], the value of an empirical $E_{0}$ is probably good to only 10 or 15 percent. Hence, agreement with eqs (10) and (11) to only about 10 to 15 percent is to be expected. Nevertheless, this is a significant improvement over a pure trial and error method. Furthermore, as more exposures are taken $E_{0}$ values can be refined.

In the transmission method, there appears to be a definite upper limit on $\left(\mu_{s} x_{s}\right)$ in order to obtain any pattern [3]. This limit is about $\mu_{s} x_{s}=10$. It is therefore recommended that eq (6) not be employed with values of $\mu_{s} x_{s}>10$.

The photographs used to determine the $E_{\mathrm{I}}$ values were prepared while one of the authors (HY) was a guest of the Massachusetts Institute Technology assigned to the laboratory of Prof. R. E. Ogilvie.

\section{References}

[1] K. Lonsdale, Divergent-beam x-Ray photography of crystals, Phil Trans Roy. Soc. (London) A-240, 219 (1947).

[2] T. Imura, Study of the deformation of single crystals by divergent x-ray beams, Bull. Naniwa Univ. A-2, 51 (1954).

[3] H. Yakowitz, Approximate optimum sample thickness for transmission Kossel photographs, to be published.

[4] D. M. Poole and P. M. Thomas, Quantitative electronprobe microanalysis, U.K. Atomic Energy Auth. Rept. AERE-R-3815, 16 pp. (1961)

[5] D. B. Brown and R. E. Ogilvie, Efficiency of production of characteristic x-radiation from pure elements bombarded by electrons, J. Appl. Phys. 35, 309 (1964).

[6] M. Green and V. E. Cosslett, The efficiency of production of characteristic x-radiation in thick targets of a pure element, Proc. Phys. Soc. (London) 78, 1206 (1961).

[7] M. Green, The efficiency of characteristic x-radiation, Thesis to Cambridge University, 246 pp. (1962).

[8] Standard Mathematical Tables, ed. C. D. Hodgman, 10th ed., Chemical Rubber Publishing Company, Cleveland, Ohio (1954), p. 340.

[9] N. P. Il'in, On the possibility of quantitative analysis by means of x-ray spectra without the use of standards, Izvest. Akad. Nauk S.S.S.R. Ser. Fiz. 25, No. 8, 929 (1961). Translated in Bull. Acad. Sci. U.S.S.R. Phys. Ser. 25, No. 8, 940 (1962).

[10] H. Yakowitz and J. R. Cuthill, A survey of correction procedures for electron probe microanalysis data, unpublished.

[11] L. S. Birks, Calculation of x-ray intensities from electron probe specimens, J. Appl. Phys. 32, 387 (1961).

[12] R. Castaing, Electron probe microanalysis, Adv. Electron and Electron Phys. 13, 317 (1960)

[13] M. Devin Quoted by J. Philbert in The Castaing "microsonde" in metallurgical and mineralogical research, J. Inst. Metals 90, 241 (1962).

[14] D. B. Wittry, Notes from a special course on electron probe microanalysis, Univ. Southern Calif. 1962.

[15] H. Yakowitz, Work performed at MIT on Fe-3 w/o Si alloys. See ref. [18] for actual photographs.

[16] W. G. Morris and R. E. Ogilvie, Kossel studies of iron whiskers, Air Force Materials Laboratory Rept. RTD-TDR-63-4198, 27 pp. (1964).

[17] P. Gielen, Kossel line studies in cubic crystals, S.M. Thesis to MIT, $48 \mathrm{pp}$. (1964).

[18] P. Gielen, Private communication, 1964. See P. Gielen, H. Yakowitz, D. Ganow and R. E. Ogilvie, Evaluation of Kossel microdiffraction procedures: The cubic case, J. Appl. Phys. 36, 773 (1965) for actual photograph.

[19] Applied Research Laboratories, The 40350 Kossel Line Camera, Special Announcement (1964).

(Paper 69C3-201) 